\title{
GENERATING FUNCTIONS FOR PRODUCTS OF RECURSIVE SEQUENCES
}

\author{
BY \\ DAVID ZEITLIN
}

1. Introduction. Recently, the author [1] gave an application of the following well-known result $[2$, p. 27]:

Let $Y_{0}, Y_{1}, \ldots, Y_{N}$ be arbitrary real numbers, and let $Y_{n}, n=0,1, \ldots$, satisfy a homogeneous, linear difference equation of order $N+1$ with real, constant coefficients:

$$
\sum_{r=0}^{N+1} a_{r} Y_{n+N+1-r}=0 \quad\left(a_{0} a_{N+1} \neq 0\right) .
$$

Then the generating function of $Y_{n}$ is given by

$$
\sum_{n=0}^{\infty} Y_{n} x^{n}=\sum_{j=0}^{N}\left[\sum_{r=0}^{j} a_{r} Y_{j-r}\right] x^{j} / \sum_{r=0}^{N+1} a_{r} x^{r} \quad(N=0,1, \ldots) .
$$

The series in (1.2) converges for $|x|<|\lambda|$, where $\lambda$ is the root of $\sum_{r=0}^{N+1} a_{r} x^{r}$ $=0$ with the smallest absolute value.

One purpose of this paper is to show how (1.2) may be applied to obtain generalizations of results recently obtained by Carlitz [3], who gave closed forms (necessitating two separate proofs) for

$$
\begin{aligned}
& \sum_{n=0}^{\infty} u_{n}^{k} x^{n} \equiv U_{k}(x) / D_{k}(x), \\
& \sum_{n=0}^{\infty} v_{n}^{k} x^{n} \equiv V_{k}(x) / D_{k}(x),
\end{aligned}
$$

where the numbers $u_{n}$ and $v_{n}[3$, pp. 521-522, 529-533] are defined by the sequences

$$
\begin{array}{llll}
u_{0}=1, & u_{1}=p, & u_{n}=p u_{n-1}-q u_{n-2} & (n=2,3, \cdots), \\
v_{0}=2, & v_{1}=p, & v_{n}=p v_{n-1}-q v_{n-2} & (n=2,3, \cdots),
\end{array}
$$

with $p^{2}-4 q \neq 0$. In (1.3a) and (1.3b) (which are the generating functions for the $k$ th power of two special second order sequences), $U_{k}(x)$ and $V_{k}(x)$ are polynomials in $x$ of degree $\leqq k$ for $k \geqq 1$, and $D_{k}(x)$ is a polynomial in $x$ of degree $k+1$. It should be pointed out that the desired form for $D_{k}(x)$ $[3$, p. $530,(6.5)]$ in terms of $a_{r}$ (i.e., our (2.4) with $m=1$ ) is given, with a

Received by the editors November 20, 1963 and, in revised form, April 6, 1964. 
different proof, in the joint paper by Jarden and Motzkin [4, Theorem 1]. An English translation of [4], which is in Hebrew, appears in [5, pp. 42-45]. Riordan [6] gives a recurrence relation for the sum function in 1.3a), where $u_{n}$ is the Fibonacci sequence. In [3], recurrence relations for the sum functions in (1.3a) and (1.3b) are also given.

In this paper, relations (1.1) and (1.2) are applied to establish five theorems on generating functions for products of recursive sequences.

Theorem 1, a generalization of (1.3a) and (1.3b), gives a closed form for

$$
\sum_{n=0}^{\infty}\left[\prod_{s=1}^{k} w_{m n+i_{s}}\right] x^{n} \quad(k, m=1,2, \cdots),
$$

where $w_{n}=p w_{n-1}-q w_{n-2}, \quad p^{2}-4 q \neq 0 \quad(n=2,3, \cdots)$, with $w_{0}$ and $w_{1}$ arbitrary real numbers, and $i_{s}, s=1,2, \cdots, k$, are positive integers or zero. An identity for generalized Fibonacci numbers, (3.1), as well as Fibonacci identities (3.9) and (3.10) are obtained as applications of (2.3) in Theorem 1. The generating functions in (4.2) and (4.3) of Theorem 2 are generalizations, respectively, of the generating functions in (2.5) and (2.6) of Theorem 1 for the case $p=0$.

Theorem 3 gives a closed form for

$$
\sum_{n=0}^{\infty}\left[\prod_{s=1}^{k} y_{m_{s} n+i_{s}}\right] x^{n} \quad(k=1,2, \cdots),
$$

where $y_{n}=\left(\sum_{j=1}^{N+1} C_{j} n^{j-1}\right) R^{n} \quad(n=0,1, \cdots)$, with $R \neq 0$ a real number, and $m_{s} \neq 0, s=1,2, \cdots, k$, are positive integers. Several applications of Theorem 3 are given in $\$ 6$, which includes binomial identities, an identity for the generalized hypergeometric series, and two identities on generalized Eulerian numbers.

Theorem 4, as a generalization of Theorem 3, gives a closed form for

$$
\sum_{n=0}^{\infty}\left[\prod_{i=1}^{t} \prod_{s=1}^{k_{i}} y_{m_{s}^{(i)} n+b_{s}^{(i)}}^{(i)}\right] x^{n} \quad(t=1,2, \cdots),
$$

where $y_{n}^{(i)}=\left(\sum_{j=1}^{N_{i}+1} C_{j}^{i} n^{j-1}\right) R_{i}^{n} \quad(n=0,1, \cdots)$, with arbitrary real numbers $R_{i} \neq 0, \quad i=1,2, \cdots, t$, and $m_{s}^{(i)} \neq 0, \quad b_{s}^{(i)} \geqq 0, \quad s=1,2, \cdots, k_{i}, \quad i=1,2, \cdots, t$, are positive integers.

Theorem 5 gives a closed form for

$$
\sum_{n=0}^{\infty} w_{m n+i_{1}}\left[\prod_{i=1}^{t} \prod_{s=1}^{k_{i}} y_{m_{s}(i)}^{(i)} b_{s}^{(i)}\right] x^{n} \quad(m=1,2, \cdots),
$$

which includes $\sum_{n=0}^{\infty} w_{m n+i_{1}} n^{N} x^{n}$ as a special case.

In $\$ 10,(6.2)$, which is a special case of Theorem 3 , is applied together with a theorem of Gould [7] on a binomial series transformation to illus- 
trate a general method for obtaining summation identities involving binomial coefficients.

The author wishes to thank the referee for his many helpful suggestions.

2. Products of equally paced subsequences of a recursive sequence of order two.

THEOREM 1. Let $w_{0}, w_{1}, p$, and $q$ be arbitrary real numbers, and define

$$
w_{n}=p w_{n-1}-q w_{n-2}, \quad p^{2}-4 q \neq 0 \quad(n=2,3, \cdots) .
$$

We will set $u_{n} \equiv w_{n}$ if $w_{0}=1, w_{1}=p$, and $v_{n} \equiv w_{n}$ if $w_{0}=2, w_{1}=p$ (see (1.4), (1.5)). Let $m=1,2, \cdots$, and define

$$
W(n, k, m) \equiv \prod_{s=1}^{k} w_{m n+i_{s}} \quad(k=1,2, \cdots ; n=0,1, \cdots),
$$

where $i_{s}, s=1,2, \cdots, k$, are positive integers or zero. Then,

(i) for $p q \neq 0, m=1,2,3, \cdots$, we have

$$
\sum_{n=0}^{\infty} W(n, k, m) x^{n}=\sum_{j=0}^{k}\left[\sum_{r=0}^{j} a_{r} W(j-r, k, m)\right] x^{j} / \sum_{r=0}^{k+1} a_{r} x^{r},
$$

where $a_{0}=1$ and

$$
a_{r}=(-1)^{r} q^{m r(r-1) / 2}\left[\frac{u_{m(k+1)-1} u_{m k-1} \cdots u_{m(k-r+2)-1}}{u_{m-1} u_{2 m-1} \cdots u_{r m-1}}\right]
$$

$$
(r=1,2, \cdots, k+1) \text {. }
$$

The series in (2.3) converges for $|x|<|\lambda|$, where $\lambda$ is the root of $a_{k+1} x^{k+1}$ $+\cdots+a_{1} x+a_{0}=0$ with the smallest absolute value;

(ii) for $p=0, m=1,3,5, \cdots$, and $|x|<|q|^{-m k / 2}$, we have

$$
\sum_{n=0}^{\infty} W(n, k, m) x^{n}=[W(0, k, m)+W(1, k, m) x] /\left[1+(-1)^{k+1} q^{m k} x^{2}\right] ;
$$

(iii) for $p=0, m=2,4,6, \cdots$, and $|x|<|q|^{-m k / 2}$, we have

$$
\sum_{n=0}^{\infty} W(n, k, m) x^{n}=W(0, k, m) /\left[1-(-q)^{m k / 2} x\right]
$$

Proof. (i) Define (see (1.1), (1.2))

$$
g(x) \equiv \sum_{r=0}^{N+1} a_{r} x^{N+1-r}, \quad h(x) \equiv x^{N+1} g(1 / x)=\sum_{r=0}^{N+1} a_{r} x^{r}
$$

It is well known that the roots of the characteristic equation, $g(x)=0$, determine the nature of the general solution to (1.1), which involves $N+1$ arbitrary constants.

Let $C_{j}^{*}, C_{j_{s}}^{* *}, j=1,2$, and $C_{0}, C_{s}, s=1, \cdots, k$, denote arbitrary constants. If $\alpha \neq \beta$ denote the roots of $x^{2}-p x+q=0$ (see (2.1)), then $w_{n}=C_{1}^{*} \alpha^{n}$ 
$+C_{2}^{*} \beta^{n}$ and $w_{m n+i_{s}}=C_{1 i_{s}}^{* *} \alpha^{m n}+C_{2 i_{s}}^{* *} \beta^{m n}$. Observing that

$$
W(n, k, m)=\sum_{s=0}^{k} C_{s}\left[\alpha^{m(k-s)} \beta^{m s}\right]^{n} \quad(n=0,1, \cdots),
$$

we now conclude that $W(n, k, m)$ satisfies a homogeneous, linear difference equation of order $k+1$ with real, constant coefficients, and that $\alpha^{m(k-s)} \beta^{m s}$, $s=0,1, \cdots, k$, are the distinct roots of the corresponding characteristic equation. Thus, $Y_{n} \equiv W(n, k, m)$ satisfies (1.1) with $N=k$, and from (2.7), we have

$$
\begin{aligned}
& g(x) \equiv \prod_{s=0}^{k}\left[x-\alpha^{m(k-s)} \beta^{m s}\right] \quad\left(a_{0}=1\right), \\
& h(x) \equiv \prod_{s=0}^{k}\left[1-\alpha^{m(k-s)} \beta^{m s} x\right] \equiv \sum_{r=0}^{k+1} a_{r} x^{r}
\end{aligned}
$$

Replacing $Y_{n}$ in (1.2) by $W(n, k, m)$ and setting $N=k$, we obtain (2.3), where the $a_{r}$, as given by (2.4), are defined by (2.10). (From [3, p. 530, (6.3), (6.4), and (6.5)], we note that $D_{k}(x) \equiv h(x)$ for $m=1$. The $a_{r}$, as given by (2.4), are obtained by a modification of the proof for (6.5), i.e., in (6.4) we replace $z$ by $(\beta / \alpha)^{m}$ and $x$ by $\alpha^{m k} x$.)

It may be of interest to note that (2.9) may be written as

$$
\begin{aligned}
\prod_{s=0}^{k}\left[x-\alpha^{m(k-s)} \beta^{m s}\right] & (k=1,3,5, \cdots) ; \\
& \equiv \begin{cases}\prod_{j=0}^{(k-1) / 2}\left[x^{2}-q^{m j} v_{m(k-2 j)} x+q^{m k}\right] & (k=2,4,6, \ldots) . \\
\left(x-q^{m k / 2}\right) \prod_{j=0}^{(k-2) / 2}\left[x^{2}-q^{m j} v_{m(k-2 j)} x+q^{m k}\right] & \end{cases}
\end{aligned}
$$

To see this, let $R_{s}=\alpha^{m(k-s)} \beta^{m s}, s=0,1, \cdots, k$. If $k=1,3,5, \cdots$, we have an even number of roots, $R_{s}$, and thus $(k+1) / 2$ pairs, $\left[\left(x-R_{j}\right)\left(x-R_{k-j}\right)\right]$, $j=0,1, \cdots,(k-1) / 2$. Since $\alpha \beta=q, \quad v_{n}=\alpha^{n}+\beta^{n}, \quad n=0,1, \cdots$, we have $R_{j}+R_{k-j}=q^{m j} v_{m(k-2 j)}$ and $R_{j} R_{k-j}=q^{m k}$.

If $k=2,4,6, \cdots$, we have an odd number of roots, $R_{s}$, and thus $k / 2$ pairs, $\quad\left[\left(x-R_{j}\right)\left(x-R_{k-j}\right)\right], j=0,1, \cdots,(k-2) / 2$. The linear term, $\left(x-R_{k / 2}\right) \equiv\left(x-q^{m k / 2}\right)$, accounts for the unpaired root, i.e., the middle root, $R_{k / 2}$.

(ii) If $p=0$, then $\beta=-\alpha$, and (2.8) simplifies to $W(n, k, m)=\sum_{s=0}^{k}$ $C_{s}\left[(-1)^{m s} \alpha^{m k}\right]^{n}$. Since $m$ is odd, $Y_{n} \equiv W(n, k, m)=C_{1}^{*}\left(\alpha^{m k}\right)^{n}+C_{2}^{*}\left(-\alpha^{m k}\right)^{n}$ satisfies (1.1) with $N=1$. Since $q=-\alpha^{2}$, we have $q^{m k}=(-1)^{k} \alpha^{2 m k}$, and from $(2.7), h(x) \equiv\left(1-\alpha^{m k} x\right)\left(1+\alpha^{m k} x\right)=1+(-1)^{k+1} q^{m k} x^{2}$, where $a_{0}=1$, $a_{1}=0$, and $a_{2}=(-1)^{k+1} q^{m k}$. Thus, (1.2) yields (2.5) for $N=1$. 
(iii) Since $p=0$ and $m$ is even, (2.8) becomes $Y_{n} \equiv W(n, k, m)=C_{1}^{*}\left(\alpha^{m k}\right)^{n}$, which satisfies (1.1) with $N=0$. Noting that $h(x) \equiv 1-\alpha^{m k} x=1$ $-(-q)^{m k / 2} x$, (1.2) yields (2.6) for $N=0$.

3. Remarks. Application to Fibonacci sequences. If $m=1, i_{s}=0, s=1,2$, $\cdots, k$, then (see (2.2)) $W(n, k, 1) \equiv u_{n}^{k}$ for $w_{0}=1$ and $w_{1}=p$, and for $p q$ $\neq 0$, (2.3) yields (1.3a); but if $w_{0}=2$ and $w_{1}=p$, then $W(n, k, 1) \equiv v_{n}^{k}$, and for $p q \neq 0$, (2.3) yields (1.3b).

If $p=0$, the sum functions of (1.3a) and (1.3b), for $k>1$ are more cumbersome to evaluate than (2.5), and, indeed, for $m=1$ and $i_{s} \equiv 0$ in (2.2) with the proper choices of $w_{0}$ and $w_{1}, U_{k}(x) / D_{k}(x)$ and $V_{k}(x) / D_{k}(x)$ must reduce to the corresponding right-hand side of (2.5).

Consider the generalized Fibonacci sequence, $H_{n}$, where $H_{0}=b, H_{1}=c$, and $H_{n+2}=H_{n+1}+H_{n}, n=0,1, \ldots$ As an application of (2.3) and (2.4), we will show that

$$
\begin{aligned}
H_{n} H_{n+1} H_{n+3} H_{n+4} & =H_{n+2}^{4}-\left(H_{2}^{4}-H_{0} H_{1} H_{3} H_{4}\right) \\
& =H_{n+2}^{4}-\left(b^{4}+2 b^{3} c-b^{2} c^{2}-2 b c^{3}+c^{4}\right)
\end{aligned}
$$

for $n=0,1, \ldots$ If $b=2, c=1$, then $H_{n} \equiv L_{n}$, the Lucas sequence, and (3.1) simplifies to

$$
L_{n} L_{n+1} L_{n+3} L_{n+4}=L_{n+2}^{4}-25 \quad(n=0,1, \ldots) ;
$$

if $b=0, c=1$, then $H_{n} \equiv F_{n}$, the standard Fibonacci sequence, and (3.1) simplifies to

$$
F_{n} F_{n+1} F_{n+3} F_{n+4}=F_{n+2}^{4}-1 \quad(n=0,1, \ldots) .
$$

In $[8$, p. 401], it is noted that (3.3) we stated by E. Gelin (1880) and proved by E. Cèsaro (1880). Closed forms for

$$
\sum_{n=0}^{N} H_{n} H_{n+1} H_{n+3} H_{n+4} \text { and } \sum_{n=0}^{N}(-1)^{n} H_{n} H_{n+1} H_{n+3} H_{n+4}
$$

may be obtained from (3.1) by a method of summation identical to that used on (3.3) by the author [9].

To prove (3.1), set $p=-q=1$ in $(2.1)$, and note that when $W(n, 4,1)$ $\equiv H_{n+2}^{4}$ or $H_{n} H_{n+1} H_{n+3} H_{n+4}$, the denominator in (2.3) is given by $1-5 x$ $-15 x^{2}+15 x^{3}+5 x^{4}-x^{5} \equiv(1-x)\left(x^{4}-4 x^{3}-19 x^{2}-4 x+1\right)$. The values, $a_{1}=-5, a_{2}=-15, a_{3}=15, a_{4}=5$, and $a_{5}=-1$, were calculated from (2.4). Omitting the cumbersome algebra, we obtain from (2.3)

$$
\begin{aligned}
\sum_{n=0}^{\infty}\left[H_{n+2}^{4}-H_{n} H_{n+1} H_{n+3} H_{n+4}\right] x^{n} & =\left(H_{2}^{4}-H_{0} H_{1} H_{3} H_{4}\right) /(1-x) \\
& =\sum_{n=0}^{\infty}\left(H_{2}^{4}-H_{0} H_{1} H_{3} H_{4}\right) x^{n} .
\end{aligned}
$$


If we equate coefficients of $x^{n}$ in (3.4), we obtain (3.1).

Results obtained from (2.3) may lead to summation identities. For example, using (2.3) and (2.4), we find that

$$
\sum_{n=0}^{\infty} F_{n} F_{n+1} F_{n+2} x^{n}=(2 x) /\left(1-3 x-6 x^{2}+3 x^{3}+x^{4}\right),
$$

$$
\sum_{n=0}^{\infty} F_{n+1}^{2} F_{n+2} x^{n}=(1-x) /\left(1-3 x-6 x^{2}+3 x^{3}+x^{4}\right) .
$$

Addition of (3.5) and (3.6) gives

$$
\sum_{n=0}^{\infty} F_{n+1} F_{n+2}^{2} x^{n}=(1+x) /\left(1-3 x-6 x^{2}+3 x^{3}+x^{4}\right) ;
$$

addition of (3.6) and (3.7) gives

$$
\sum_{n=0}^{\infty} F_{n+1} F_{n+2} F_{n+3} x^{n}=2 /\left(1-3 x-6 x^{2}+3 x^{3}+x^{4}\right) .
$$

Since $1-3 x-6 x^{2}+3 x^{3}+x^{4} \equiv\left(1-4 x-x^{2}\right)\left(1+x-x^{2}\right)$, the series in (3.5), (3.6), (3.7), and (3.8) all converge for $|x|<(\sqrt{ } 5-2)$. If we multiply both sides of (3.6) by $2 /(1-x)$ and note (3.8), we obtain the following identity:

$$
2 \sum_{j=0}^{n} F_{j+1}^{2} F_{j+2}=F_{n+1} F_{n+2} F_{n+3} \quad(n=0,1, \cdots) .
$$

If we multiply both sides of (3.7) by $2 /(1+x)$ and note (3.8), we obtain the following identity:

$$
2 \sum_{j=0}^{n}(-1)^{j} F_{j+1} F_{j+2}^{2}=(-1)^{n} F_{n+1} F_{n+2} F_{n+3} \quad(n=0,1, \cdots) .
$$

4. Unequally paced subsequences. A generalization of Theorem 1 is called for if we consider (2.1) and (4.1) (instead of (2.2)), where

$$
W^{*}(n, k) \equiv \prod_{s=1}^{k} w_{m_{s} n+i_{s}} \quad(k=1,2, \cdots ; n=0,1, \ldots),
$$

and $m_{s} \neq 0, s=1,2, \cdots, k$, are positive integers.

The case $p q \neq 0$ for (2.1) is difficult, and for arbitrary $k$ in (4.1), the author was unable to give the complete set of values for $a_{r}$ as required by (1.2).

The case $p=0$ for (2.1) is readily obtained for arbitrary $k$ in (4.1), and the proof of Theorem 2 is omitted, since it is similar to that of (ii) and (iii) for Theorem 1. 
THEOREM 2. Let $W^{*}(n, k)$ satisfy (4.1), where $w_{n}$ satisfies (2.1) with $p=0, q \neq 0$. Let $M=m_{1}+m_{2}+\cdots+m_{k}$. Then,

(i) ${ }^{*}$ if at least one $m_{s}, s=1,2, \cdots, k$, is an odd integer,

$$
\sum_{n=0}^{\infty} W^{*}(n, k) x^{n}=\left[W^{*}(0, k)+W^{*}(1, k) x\right] /\left[1-(-q)^{M} x^{2}\right]
$$

$$
\left(|x|<|q|^{-M / 2}\right)
$$

(ii) ${ }^{*}$ if all the $m_{s}, s=1,2, \cdots, k$, are even integers,

$$
\sum_{n=0}^{\infty} W^{*}(n, k) x^{n}=W^{*}(0, k) /\left[1-(-q)^{M / 2} x\right] \quad\left(|x|<|q|^{-M / 2}\right) .
$$

We note that if $m_{s} \equiv m \neq 0, s=1,2, \cdots, k$, then (4.2) and (4.3), respectively, reduce to (2.5) and (2.6) of Theorem 1.

5. Subsequences of a sequence of higher order. Thus far, we have assumed that $p^{2} \neq 4 q$ in (2.1). In $\left[3\right.$, p. 535] the case $p^{2}=4 q$ requires a method of proof different from that used to obtain (1.3a) and (1.3b). We will now establish a general result which, as a special case, contains the generalization of the case $p^{2}=4 q$.

Theorem 3. Let $y_{n}, n=0,1, \cdots, N$, be arbitrary real numbers, and let $y_{n}$ be the general solution of (1.1) with

$$
a_{i}=(-R)^{i}\left(\begin{array}{c}
N+1 \\
i
\end{array}\right) \quad(i=0,1, \cdots, N+1),
$$

where $R \neq 0$ is a real number. Let $M=m_{1}+m_{2}+\cdots+m_{k}$, where $m_{s} \neq 0$, $s=1,2, \cdots, k$, are positive integers, and set

$$
Q(n, k) \equiv \prod_{s=1}^{k} y_{m_{s} n+i_{s}} \quad(k=1,2, \cdots ; n=0,1, \cdots),
$$

where $i_{s}, s=1,2, \cdots, k$, are positive integers or zero. Then, for $|x|<|R|^{-M}$ and $N=0,1, \cdots$, we have

$$
\begin{aligned}
& \left(1-R^{M} x\right)^{k N+1} \sum_{n=0}^{\infty} Q(n, k) x^{n} \\
& =\sum_{j=0}^{k N}\left[\sum_{r=0}^{j}(-1)^{r} R^{M r}\left(\begin{array}{c}
k N+1 \\
r
\end{array}\right) Q(j-r, k)\right] x^{j} .
\end{aligned}
$$

Proof. Using (2.7), we note that $g(x) \equiv(x-R)^{N+1}$ and, hence, $y_{n}$ $=\left(\sum_{j=1}^{N+1} C_{j} n^{j-1}\right) R^{n}$, where the $C_{j}, j=1,2, \cdots, N+1$, are arbitrary constants. Since

$$
y_{m_{s} n+i_{s}}=\left(\sum_{j=1}^{N+1} C_{j}^{*} n^{j-1}\right) R^{m_{s} n} \quad \text { and } \quad Q(n, k)=\left(\sum_{j=1}^{k N+1} C_{j}^{* *} n^{j-1}\right) R^{M n},
$$


where $C_{j}^{*}$ and $C_{j}^{* *}$ are again arbitrary constants, we conclude that $Q(n, k)$ satisfies a homogeneous, linear difference equation of order $k N+1$ with real, constant coefficients, whose characteristic equation is $g^{*}(x) \equiv\left(x-R^{M}\right)^{k N+1}$ $=0$. Thus, $Q(n, k) \equiv Y_{n}$ satisfies

$$
\begin{aligned}
\sum_{r=0}^{k N+1} a_{r}^{*} Y_{n+k N+1-r}=0, \quad \text { where } a_{r}^{*}=(-1)^{r} R^{M r}\left(\begin{array}{c}
k N+1 \\
r
\end{array}\right), \\
r=0,1, \cdots, k N+1 .
\end{aligned}
$$

An application of (1.2) (in which $N$ is replaced by $k N$ ) yields our result, (5.3).

6. Binomial and other identities. The generalizations of the cases (1.3a) and (1.3b) for (2.1) with $p^{2}=4 q$ (as given in $[3$, p. 535]) are obtained from (5.3) when $N=1$.

Setting $R=1, y_{n} \equiv n^{N}, n=0,1, \cdots, m_{s} \equiv m$ and $i_{s} \equiv b, \quad s=1,2, \cdots, k$, and $k N=c$ in (5.3), we obtain

$$
\begin{aligned}
(1-x)^{c+1} & \sum_{n=0}^{\infty}(m n+b)^{c} x^{n} \\
= & \sum_{j=0}^{c}\left[\sum_{r=0}^{j}(-1)^{r}\left(\begin{array}{c}
c+1 \\
r
\end{array}\right)(m(j-r)+b)^{c}\right] x^{j} \quad(|x|<1) .
\end{aligned}
$$

A less elegant version of (6.1) may be found in $[10$, p. 99, (171), .., (175) ]. The derivation of (6.1) for $m=1$ and $b=0$ was given previously by the author [1], as well as by others (see [1] for references).

For $N=0,1, \ldots$, the following identity (valid for $|x|<1$ )

$$
\begin{aligned}
& (1-x)^{k N+1} \sum_{n=0}^{\infty}\left[\prod_{i=1}^{\sigma}\left(\begin{array}{c}
c_{i} n+k_{i} \\
k_{i}
\end{array}\right)^{N}\right] x^{n} \\
& =\sum_{j=0}^{k N}\left[\sum_{r=0}^{j}(-1)^{r}\left(\begin{array}{c}
k N+1 \\
r
\end{array}\right) \prod_{i=1}^{\infty}\left(\begin{array}{c}
c_{i}(j-r)+k_{i} \\
k_{i}
\end{array}\right)^{N}\right] x^{j},
\end{aligned}
$$

where $c_{i}, i=1,2, \cdots, \sigma$, are positive integers, and $k=k_{1}+k_{2}+\cdots+k_{\sigma}$, with $k_{i}=1,2, \cdots, i=1,2, \cdots, \sigma$, is obtained from (5.3) by setting $R=1$, $y_{n} \equiv n^{N}, n=0,1, \cdots$, and

$$
\begin{array}{ll}
m_{s} \equiv c_{1}, i_{s} \equiv s, & s=1,2, \cdots, k_{1} ; \\
m_{s} \equiv c_{2}, i_{s} \equiv s-k_{1}, & s=k_{1}+1, k_{1}+2, \cdots, k_{1}+k_{2} ;
\end{array}
$$

$$
m_{s} \equiv c_{\sigma}, i_{s} \equiv s-\sum_{i=1}^{\sigma-1} k_{i}, s=\left[\sum_{i=1}^{o-1} k_{i}\right]+1,\left[\sum_{i=1}^{\sigma-1} k_{i}\right]+2, \cdots, k
$$


Generalized Eulerian numbers, $A_{k, s,}^{(i)}$, whose properties are given in [11, p. 240, (9)], occur in (6.2) when $\sigma=1$ (i.e., $k_{1}=k$ ) and $c_{1}=1$, where

$$
A_{N, j+1}^{(k)}=\sum_{r=0}^{j}(-1)^{r}\left(\begin{array}{c}
k N+1 \\
r
\end{array}\right)\left(\begin{array}{c}
j-r+k \\
k
\end{array}\right)^{N} \quad(N, k=1,2, \cdots)
$$

Recently, Marx [12] pointed out that the following identity,

$$
\begin{aligned}
& \sum_{n=0}^{\infty}\left(\begin{array}{c}
n+a-1 \\
a-1
\end{array}\right)\left(\begin{array}{c}
n+b-1 \\
b-1
\end{array}\right) x^{n} \\
& =(1-x)^{1-a-b} \sum_{j=0}^{a-1}\left(\begin{array}{c}
a-1 \\
j
\end{array}\right)\left(\begin{array}{c}
b-1 \\
j
\end{array}\right) x^{j} \quad(|x|<1),
\end{aligned}
$$

is obtained by an application of a well-known transformation formula for the hypergeometric series $[13$, p. $105,(1),(2)]$, i.e.,

$$
F(a, b ; 1 ; x)=(1-x)^{1-a-b} F(1-a, 1-b ; 1 ; x) .
$$

But from (6.2), with $N=1, \sigma=2, c_{1}=c_{2}=1, k_{1}=a-1$, and $k_{2}=b-1$, we obtain

$$
L(x)=(1-x)^{1-a-b}
$$

$$
\cdot \sum_{j=0}^{a+b-2}\left[\sum_{r=0}^{j}(-1)^{r}\left(\begin{array}{c}
a+b-1 \\
r
\end{array}\right)\left(\begin{array}{c}
j-r+a-1 \\
a-1
\end{array}\right)\left(\begin{array}{c}
j-r+b-1 \\
b-1
\end{array}\right)\right] x^{j}
$$

where $L(x)$ denotes the left-hand side of (6.5). Comparing (6.5) and (6.6), we conclude that

$$
\begin{gathered}
\sum_{r=0}^{j}(-1)^{r}\left(\begin{array}{c}
a+b-1 \\
r
\end{array}\right)\left(\begin{array}{c}
j-r+a-1 \\
a-1
\end{array}\right)\left(\begin{array}{c}
j-r+b-1 \\
b-1
\end{array}\right) \\
\equiv\left(\begin{array}{c}
a-1 \\
j
\end{array}\right)\left(\begin{array}{c}
b-1 \\
j
\end{array}\right) .
\end{gathered}
$$

Setting $a=b=n$ in (6.7), we obtain, noting (6.4),

$$
A_{2, j+1}^{(n-1)} \equiv\left(\begin{array}{c}
n-1 \\
j
\end{array}\right)^{2} \equiv \sum_{r=0}^{j}(-1)^{r}\left(\begin{array}{c}
2 n-1 \\
r
\end{array}\right)\left(\begin{array}{c}
j-r+n-1 \\
n-1
\end{array}\right)^{2} .
$$

Recalling the definition of the generalized hypergeometric series [13, p. 182],

$$
{ }_{p} F_{q}\left[\begin{array}{c}
a_{1}, \cdots, a_{p} ; x \\
\rho_{1}, \cdots, \rho_{q}
\end{array}\right]=\sum_{n=0}^{\infty} \frac{\left(a_{1}\right)_{n} \cdots\left(a_{p}\right)_{n} x^{n}}{\left(\rho_{1}\right)_{n} \cdots\left(\rho_{q}\right)_{n} n !},
$$

where $(a)_{0} \equiv 1, \quad(a)_{n}=a(a+1) \cdots(a+n-1) \quad(n=1,2, \cdots)$, we note that (6.2), with $N=1, c_{i} \equiv 1$ and $k_{i}=a_{i}-1, i=1,2, \cdots, \sigma \equiv q+1$, may written as 


$$
\begin{aligned}
& (1-x)^{T(q)}{ }_{q+1} F_{q}\left[\begin{array}{c}
a_{1}, a_{2}, \cdots, a_{q+1} ; x \\
1,1, \cdots, 1
\end{array}\right] \\
& =\sum_{j=0}^{T(q)-1}\left[\sum_{r=0}^{j}(-1)^{r}\left(\begin{array}{c}
T(q) \\
r
\end{array}\right) \prod_{i=1}^{q+1}\left(\begin{array}{c}
j-r+a_{i}-1 \\
a_{i}-1
\end{array}\right)\right] x^{j} \quad(|x|<1),
\end{aligned}
$$

where $T(q)=-q+\sum_{i=1}^{q+1} a_{i}$. If we set $a_{1}=a_{2}=\cdots=a_{q+1}$, the series in (6.9) is then well-poised [13, p. 188], since $1+a_{1}=1+a_{2}=\ldots=1$ $+a_{q+1}$. For the well-poised case, we observe (again) that the right-hand side of (6.9), with $a_{i} \equiv a, i=1,2, \cdots, q+1$, is expressible in terms of $A_{q+1, j+1}^{(a-1)}$ (see (6.4)).

From (6.2), with $\sigma=N=1, k_{1}=k$, and $c_{1}=1$, we obtain (noting (6.4))

$$
(1-x)^{k+1} \sum_{n=0}^{\infty}\left(\begin{array}{c}
n+k \\
k
\end{array}\right) x^{n}=\sum_{j=0}^{k} A_{1, j+1}^{(k)} x^{j} \quad(|x|<1) .
$$

But $[2$, p. $30,(4)]$

$$
(1-x)^{k+1} \sum_{n=0}^{\infty}\left(\begin{array}{c}
n+k \\
k
\end{array}\right) x^{n}=1 \quad(|x|<1) .
$$

Comparing (6.10) and (6.11), we conclude that

$$
A_{1, j+1}^{(k)} \equiv \sum_{r=0}^{j}(-1)^{r}\left(\begin{array}{c}
k+1 \\
r
\end{array}\right)\left(\begin{array}{c}
j-r+k \\
k
\end{array}\right)=0 \quad(j=1,2, \cdots, k)
$$

I want to thank Professor H. W. Gould for the following comments and references concerning (6.7). Since (6.7) is a polynomial identity for integers $a$ and $b$, it is also valid for all real $a$ and $b$. Thus, (6.7), with $a=x+1$, $b=y+1$, and $j=n$, under the variable change $r=n-k$, becomes

$$
\sum_{k=0}^{n}(-1)^{n-k}\left(\begin{array}{c}
x+y+1 \\
n-k
\end{array}\right)\left(\begin{array}{c}
x+k \\
k
\end{array}\right)\left(\begin{array}{c}
y+k \\
k
\end{array}\right)=\left(\begin{array}{l}
x \\
n
\end{array}\right)\left(\begin{array}{l}
y \\
n
\end{array}\right)
$$

(all real $x, y)$.

Using elementary transformations on the binomial coefficients, Gould (in a letter to the author) shows that (6.13) is equivalent to an indentity of Surányi [14]. Carlitz [15], in a review of [14], points out that Surányi's identity is a special case of Saalschütz's formula $[13$, p. 66, (30)].

\section{Subsequences of distinct sequences.}

Theorem 4. Let $N_{i}, i=1,2, \cdots, t$, be positive integers or zero, and for $n=0,1, \cdots$, let $y_{n}^{(i)}, i=1,2, \cdots, t$, be the general solution of

$$
\sum_{j=0}^{N_{i}+1} a_{j}^{(i)} y_{n+N_{i}+1-j}^{(i)}=0 \quad\left(a_{0}^{(i)} a_{N_{i}+1}^{(i)} \neq 0\right),
$$

with 


$$
a_{j}^{(i)}=\left(-R_{i}\right)^{j}\left(\begin{array}{c}
N_{i}+1 \\
j
\end{array}\right) \quad\left(j=0,1, \cdots, N_{i}+1\right),
$$

where $R_{i} \neq 0, \quad i=1,2, \cdots, t$, are real numbers. Let $M_{i}=m_{1}^{(i)}+m_{2}^{(i)}+\cdots$ $+m_{k_{i}}^{(i)}$, where $m_{s}^{(i)} \neq 0, s=1,2, \cdots, k_{i}$, and $k_{i}, i=1,2, \cdots, t$, are positive integers, and set

$$
Q\left(n, k_{i}\right)=\prod_{s=1}^{k_{i}} y_{m_{s}^{(i)} n+b_{s}^{(i)}}^{(i)} \quad(i=1,2, \cdots, t ; n=0,1, \cdots)
$$

$$
Q^{*}(n)=\prod_{i=1}^{t} Q\left(n, k_{i}\right) \quad(t=1,2, \cdots)
$$

where $b_{s}^{(i)}, s=1,2, \cdots, k_{i}, i=1,2, \cdots, t$, are positive integers or zero. Let $R=\prod_{i=1}^{t} R_{i}^{M}$ and $N=\sum_{i=1}^{t} k_{i} N_{i}$. Then, for $|x|<|R|^{-1}$, we have

$$
(1-R x)^{N+1} \sum_{n=0}^{\infty} Q^{*}(n) x^{n}=\sum_{j=0}^{N}\left[\sum_{r=0}^{j}(-1)^{r} R^{r}\left(\begin{array}{c}
N+1 \\
r
\end{array}\right) Q^{*}(j-r)\right] x^{j}
$$

Proof. Noting the proof of Theorem 3, we observe that

$$
Q\left(n, k_{i}\right)=\left(\sum_{j=1}^{k_{i} N_{i}+1} C_{j} n^{j-1}\right) R_{i}^{M_{i} n}, \quad Q^{*}(n)=\left(\sum_{j=1}^{N+1} C_{j}^{*} n^{j-1}\right) R^{n},
$$

where $C_{j}$ and $C_{j}^{*}$ are arbitrary constants. Thus, $Q^{*}(n)$ satisfies a homogeneous, linear difference equation of order $N+1$ with real, constant coefficients, whose characteristic equation is $g(x) \equiv(x-R)^{N+1}=0$. Since $Q^{*}(n) \equiv Y_{n}$ satisfies (1.1), where

$$
a_{r}=(-1)^{r} R^{r}\left(\begin{array}{c}
N+1 \\
r
\end{array}\right), \quad r=0,1, \cdots, N+1
$$

an application of (1.2) yields (7.4).

8. Another binomial identity. We note that for $t=1,(7.4)$ reduces to (5.3). Generalizations of (6.1) and (6.2) may be obtained from (7.4). Indeed, for $i=1,2, \cdots, t$, let $R=1, \quad R_{i} \equiv 1, \quad N_{i}=0,1, \cdots, y_{n}^{(i)} \equiv n^{N_{i}}, n$ $=0,1, \cdots ; \sigma_{i}=1,2, \cdots ; k_{i}=\sum_{j=1}^{\sigma_{i}} k_{i j}$, where $\left\{k_{i j}\right\}$ and $\left\{c_{i j}\right\}, j=1,2, \cdots$, $\sigma_{i}$, are positive integers, and define (see (6.3))

$$
\begin{aligned}
& m_{s}^{(i)} \equiv c_{i 1}, b_{s}^{(i)} \equiv s, \quad s=1,2, \cdots, k_{i 1} ; \\
& m_{s}^{(i)} \equiv c_{i 2}, b_{s}^{(i)} \equiv s-k_{i 1}, \quad s=k_{i 1}+1, k_{i 1}+2, \cdots, k_{i 1}+k_{i 2} ; \\
& m_{s}^{(i)} \equiv c_{i \sigma_{i}}, b_{s}^{(i)} \equiv s-\sum_{j=1}^{\sigma_{i}-1} k_{i j}, s=\left[\sum_{j=1}^{\sigma_{i}-1} k_{i j}\right]+1,\left[\sum_{j=1}^{\sigma_{i}-1} k_{i j}\right]+2, \cdots, k_{i} .
\end{aligned}
$$


With the above definitions, (7.4) yields the following identity, where $N$ $=\sum_{i=1}^{t} N_{i} \sum_{j=1}^{\sigma_{i}} k_{i j}$ :

$$
\begin{aligned}
& (1-x)^{N+1} \sum_{n=0}^{\infty}\left[\prod_{i=1}^{t} \prod_{j=1}^{\sigma_{i}}\left(\begin{array}{c}
c_{i j} n+k_{i j} \\
k_{i j}
\end{array}\right)^{N_{i}}\right] x^{n} \\
& =\sum_{j=0}^{N}\left[\sum_{r=0}^{j}(-1)^{r}\left(\begin{array}{c}
N+1 \\
r
\end{array}\right) \prod_{i=1}^{t} \prod_{\mu=1}^{\sigma_{i}}\left(\begin{array}{c}
c_{i \mu}(j-r)+k_{i \mu} \\
k_{i \mu}
\end{array}\right)^{N_{i}}\right] x^{j} \quad(|x|<1) .
\end{aligned}
$$

\section{An additional factor taken from a sequence of order two.}

Theorem 5. Let $Q^{*}(n)$ (see (7.3)), $N$, and $R$ be defined by Theorem 4, and $w_{n}$ by (2.1), with $p q \neq 0$, of Theorem 1 . Let $m=1,2, \cdots$, and $i_{1}=0,1$, $\ldots$ Let $|\alpha|<|\beta|$, where $(x-\alpha)(x-\beta) \equiv x^{2}-p x+q$. Then, for $|x|$ $<\left(|R \| \beta|^{m}\right)^{-1}$, we have

$$
\begin{aligned}
\left(1-v_{m} R x+q^{m} R^{2} x^{2}\right)^{N+1} & \sum_{n=0}^{\infty} w_{m n+i_{1}} Q^{*}(n) x^{n} \\
= & \sum_{j=0}^{2 N+1}\left[\sum_{r=0}^{j} a_{r} w_{m(j-r)+i_{1}} Q^{*}(j-r)\right] x^{j},
\end{aligned}
$$

where

$$
a_{r}=(-R)^{r} \sum_{s=0}^{r}\left(\begin{array}{c}
N+1 \\
s
\end{array}\right)\left(\begin{array}{c}
s \\
r-s
\end{array}\right) v_{m}^{2 s-r} q^{m(r-s)} \quad(r=0,1, \cdots, 2 N+2) .
$$

Proof. From the proof of Theorem 4, we recall that

$$
Q^{*}(n)=\left(\sum_{j=1}^{N+1} C_{j}^{*} n^{j-1}\right) R^{n},
$$

and from the proof of Theorem 1, (i), we recall that $w_{m n+i_{1}}=C_{1} \alpha^{m n}+C_{2} \beta^{m n}$. Thus,

$$
\begin{aligned}
Y_{n} \equiv w_{m n+i_{1}} Q^{*}(n)=\left(\sum_{j=1}^{N+1} C_{1} C_{j}^{*} n^{j-1}\right)\left(\alpha^{m} R\right)^{n} \\
+\left(\sum_{j=1}^{N+1} C_{2} C_{j}^{*} n^{j-1}\right)\left(\beta^{m} R\right)^{n}, \quad n=0,1, \cdots,
\end{aligned}
$$

satisfies a homogeneous, linear difference equation of order $2(N+1)$ with real constant coefficients, whose characteristic equation is

$$
g(x) \equiv\left(x-\alpha^{m} R\right)^{N+1}\left(x-\beta^{m} R\right)^{N+1} \equiv\left(x^{2}-v_{m} R x+q^{m} R^{2}\right)^{N+1}=0
$$

(since $\alpha \beta=q$ and $v_{m}=\alpha^{m}+\beta^{m}$ ). Noting (2.7), we see that $\sum_{r=0}^{2 N+2} a_{r} x^{r}$ $\equiv\left(1-v_{m} R x+q^{m} R^{2} x^{2}\right)^{N+1}$, and the expression for $a_{r}$ (see (9.2)) follows 
from [2, p. 30, Example 3]. Thus, (9.1) is obtained by an application of (1.2).

It should be noted that our method of proof for Theorem 5 could also be used to obtain the generating function for $W(n, k, m) Q^{*}(n)$ (see (2.2) and (2.8), where $p q \neq 0)$. We observe that $W Q^{*}$ satisfies a homogeneous, linear difference equation of order $(k+1)(N+1)$; and if $g(x)=0$ (see (2.9)) is the characteristic equation for $W$, then $[g(x / R)]^{N+1}=0$ (i.e., a polynomial equation in $x$ of degree $(k+1)(N+1))$ is the characteristic equation for $W Q^{*}$. The desired result, obtained by an application of (1.2), is cumbersome, since the expressions for $a_{r}$ (the coefficients of the characteristic polynomial) are formidable.

10. Evaluation of sums of products of binomial coeficients. We will now show how Theorem 3 may be used in conjunction with a result of Gould to obtain identities, closely related to convolution identities. The binomial series transformation $[7,(2.1),(2.2), 3.1)]$, referred to as a Vandermonde convolution transform, is defined as follows:

Let $f(k)$ be independent of $n$ and $f(0)=1$. Set

$$
F(n)=\sum_{k=0}^{n}(-1)^{k}\left(\begin{array}{l}
n \\
k
\end{array}\right)\left(\begin{array}{c}
a+b k \\
n
\end{array}\right) f(k)
$$

Then

$$
\sum_{k=0}^{\infty}\left(\begin{array}{c}
a+b k \\
k
\end{array}\right) f(k) z^{k}=x^{a} \sum_{n=0}^{\infty}(-1)^{n} F(n)[(x-1) / x]^{n} \quad(x \neq 0),
$$

where $z=(x-1) / x^{b}$. Moreover,

$$
\left(\begin{array}{c}
a+b n \\
n
\end{array}\right) f(n)=\sum_{k=0}^{n}(-1)^{k}\left[\frac{a+b k-k}{a+b n-k}\left(\begin{array}{c}
a+b n-k \\
n-k
\end{array}\right) F(k)\right] .
$$

For our purpose, we take $b=1$. Then (10.2) may be written as

$$
(1-z)^{a} \sum_{n=0}^{\infty}\left(\begin{array}{c}
n+a \\
a
\end{array}\right) f(n) z^{n}=\sum_{n=0}^{\infty}(-1)^{n} F(n) z^{n} .
$$

For proper choices of $a$ and $f(n)$, (10.4) yields different identities (i.e., other than (10.3)) provided a closed form for the left-hand side of (10.4) is known. For simplicity, only (6.2), a special case of Theorem 3, will be used with (10.4) to illustrate the ideas involved.

For our first example, consider (6.5), which may be written as

$$
(1-z)^{a+c+1} \sum_{n=0}^{\infty}\left(\begin{array}{c}
n+a \\
a
\end{array}\right)\left(\begin{array}{c}
n+c \\
c
\end{array}\right) z^{n}=\sum_{n=0}^{a}\left(\begin{array}{c}
a \\
n
\end{array}\right)\left(\begin{array}{c}
c \\
n
\end{array}\right) z^{n} \quad(|z|<1) .
$$


Let

$$
f(k) \equiv\left(\begin{array}{c}
k+c \\
c
\end{array}\right)
$$

Multiplying both sides of $(10.4)$ by $(1-z)^{c+1}$, we obtain, upon comparison of (10.4) with (10.5),

$$
\begin{aligned}
\sum_{n=0}^{a}\left(\begin{array}{l}
a \\
n
\end{array}\right)\left(\begin{array}{l}
c \\
n
\end{array}\right) z^{n} & =(1-z)^{c+1} \sum_{n=0}^{\infty}(-1)^{n} F(n) z^{n} \\
& =\sum_{n=0}^{\infty}\left[(-1)^{n} \sum_{k=0}^{n}\left(\begin{array}{c}
c+1 \\
n-k
\end{array}\right) F(k)\right] z^{n}
\end{aligned}
$$

Upon equating coefficients in (10.6), we obtain.

$$
\left(\begin{array}{l}
a \\
n
\end{array}\right)\left(\begin{array}{l}
c \\
n
\end{array}\right)=(-1)^{n} \sum_{k=0}^{n}\left(\begin{array}{c}
c+1 \\
n-k
\end{array}\right) F(k) .
$$

With $b=1$ and our choice of $f(k),(10.1)$ and (10.3) yield, respectively,

$$
\begin{aligned}
F(n) & =\sum_{k=0}^{n}(-1)^{k}\left(\begin{array}{l}
n \\
k
\end{array}\right)\left(\begin{array}{c}
a+k \\
n
\end{array}\right)\left(\begin{array}{c}
c+k \\
c
\end{array}\right), \\
\left(\begin{array}{c}
a+n \\
n
\end{array}\right)\left(\begin{array}{c}
c+n \\
n
\end{array}\right) & =\sum_{k=0}^{n}(-1)^{k}\left(\begin{array}{c}
a+n-1-k \\
a-1
\end{array}\right) F(k) .
\end{aligned}
$$

Neither of the identities (10.7) and (10.9) seems to be an immediate consequence of the other.

It is of interest to note that with $b=a=1$, (10.1) has only two nonzero terms, i.e., when $k=n-1$ and $k=n$, with $f(k)$ arbitrary. Thus, (10.1) becomes

$$
F(n)=(-1)^{n} \Delta(n f(n-1)),
$$

where $\Delta g(n)=g(n+1)-g(n)$. Qf course, (10.4) yields (10.10) for $a=1$. Moreover, (10.3) (with $b=a=1$ ) reduces to the trivial identity, $(n+1) f(n)$ $\equiv \sum_{k=0}^{n} \Delta[k f(k-1)]$.

To avoid notational confusion, replace now $a$ in (10.5) by $p$. Then, set $a=1$ in (10.4) and define

$$
f(k) \equiv\left(\begin{array}{c}
p+k \\
p
\end{array}\right)\left(\begin{array}{c}
c+k \\
c
\end{array}\right) /(k+1) .
$$

Multiplying both sides of $(10.4)$ by $(1-z)^{p+c}$, we obtain, upon comparison of (10.4) with (10.5), 


$$
\begin{aligned}
\sum_{n=0}^{p}\left(\begin{array}{l}
p \\
n
\end{array}\right)\left(\begin{array}{l}
c \\
n
\end{array}\right) z^{n} & =(1-z)^{p+c} \sum_{n=0}^{\infty}(-1)^{n} F(n) z^{n} \\
& =\sum_{n=0}^{\infty}\left[(-1)^{n} \sum_{k=0}^{n}\left(\begin{array}{l}
p+c \\
n-k
\end{array}\right) F(k)\right] z^{n}
\end{aligned}
$$

Equating coefficients in (10.11), we obtain (recalling (10.10)),

$$
\left(\begin{array}{l}
p \\
n
\end{array}\right)\left(\begin{array}{l}
c \\
n
\end{array}\right)
$$

$$
=(-1)^{n} \sum_{k=0}^{n}(-1)^{k}\left(\begin{array}{c}
p+c \\
n-k
\end{array}\right) \Delta\left[\left(\begin{array}{c}
p+k-1 \\
p
\end{array}\right)\left(\begin{array}{c}
c+k-1 \\
c
\end{array}\right)\right],
$$

$$
\left(\begin{array}{l}
p \\
n
\end{array}\right)\left(\begin{array}{l}
c \\
n
\end{array}\right)=(-1)^{n+1} \sum_{k=0}^{n}\left(\begin{array}{c}
p+k \\
p
\end{array}\right)\left(\begin{array}{c}
c+k \\
c
\end{array}\right) \Delta\left[(-1)^{k}\left(\begin{array}{l}
p+c \\
n-k
\end{array}\right)\right],
$$

$$
\left(\begin{array}{l}
p \\
n
\end{array}\right)\left(\begin{array}{l}
c \\
n
\end{array}\right)=\sum_{k=0}^{n}(-1)^{n-k}\left(\begin{array}{c}
p+k \\
p
\end{array}\right)\left(\begin{array}{c}
c+k \\
c
\end{array}\right)\left(\begin{array}{c}
p+c+1 \\
n-k
\end{array}\right)
$$

where (10.13) is obtained from (10.12) by summation by parts, and (10.14) is obtained from (10.13), using

$$
\left(\begin{array}{c}
m \\
k-1
\end{array}\right)+\left(\begin{array}{c}
m \\
k
\end{array}\right)=\left(\begin{array}{c}
m+1 \\
k
\end{array}\right)
$$

We note that (10.14) is precisely $(6.13)$, which was previously obtained by comparing (6.5) with (6.6).

Suppose now that the expansion

$$
(1-z)^{m+1} \sum_{n=0}^{\infty} h(n) z^{n}=\sum_{j=0}^{m} c_{j} z^{j}
$$

is valid for $|z|<1$, where $h(n), n=0,1, \cdots$, are given, and the $c_{j}, j=0,1$, $\cdots, m$, are to be determined. Then, our procedure, when $a=1$ in (10.4), yields the explicit representation of $c_{j}$. Indeed, suppose

$$
(1-x)^{k N+1} \sum_{n=0}^{\infty}\left(\begin{array}{c}
n+k \\
k
\end{array}\right)^{N} x^{n}=\sum_{j=0}^{k N} c_{j} x^{j} \quad(|x|<1),
$$

where $k$ and $N$ are positive integers, and $c_{j}$ are unknown constants. Put $a=1$ and

$$
f(n) \equiv\left(\begin{array}{c}
n+k \\
k
\end{array}\right)^{N} /(n+1)
$$

in (10.4), with $z \equiv x$. Upon multiplication of both sides of (10.4) by $(1-x)^{k N}$, we obtain, in view of $(10.10)$ and $(10.15)$, 


$$
\begin{aligned}
c_{j} & =(-1)^{j} \sum_{r=0}^{j}(-1)^{r}\left(\begin{array}{c}
k N \\
j-r
\end{array}\right) \Delta\left[\left(\begin{array}{c}
r-1+k \\
k
\end{array}\right)^{N}\right] \\
& =(-1)^{j+1} \sum_{r=0}^{j}\left(\begin{array}{c}
r+k \\
k
\end{array}\right)^{N} \Delta\left[(-1)^{r}\left(\begin{array}{c}
k N \\
j-r
\end{array}\right)\right] \\
& =\sum_{r=0}^{j}(-1)^{j-r}\left(\begin{array}{c}
k N+1 \\
j-r
\end{array}\right)\left(\begin{array}{c}
r+k \\
k
\end{array}\right)^{N}=A_{N, j+1}^{(k)} \quad(\text { see (6.4)). }
\end{aligned}
$$

Our procedure, when applied to (10.4) for $a \neq 1$, will, in general, lead to new summation identities, provided the multiplier of $(10.4)$ is $(1-z)^{m}$, with $m \neq 1$. This point has already been illustrated in the derivation of (10.7). Moreover, (6.2) (with $c_{i} \neq 1$ ), when used with (10.4), furnishes new identities.

\section{REFERENCES}

1. D. Zeitlin, Two methods for the evaluation of $\sum_{k=0}^{\infty} k^{n} x^{k}$, Amer. Math. Monthly 68 (1961), 986-989.

2. C. Jordan, Calculus of finite differences, Chelsea, New York, 1960.

3. L. Carlitz, Generating functions for powers of certain sequences of numbers, Duke Math. J. 29 (1962), 521-538.

4. D. Jarden and T. Motzkin, The product of sequences with a common linear recursion formula of order 2, Riveon Lematematika 3 (1949), 25-27, 38. (Hebrew. English summary) 1958.

5. D. Jarden, Recurring sequences; a collection of papers, Riveon Lematematika, Jerusalem,

6. J. Riordan, Generating functions for powers of Fibonacci numbers, Duke Math. J. 29 (1962), 5-12.

7. H. W. Gould, $A$ series transformation for finding convolution identities, Duke Math. J. 28 (1961), 193-202.

8. L. E. Dickson, History of the theory of numbers, Vol. 1, Chelsea, New York, 1952.

9 D. Zeitlin, On identities for Fibonacci numbers, Amer. Math. Monthly 70 (1963), 987-991.

10. I. J. Schwatt, An introduction to the operations with series, Chelsea, New York, 1962.

11. L. Carlitz, Note on a paper of Shanks, Amer. Math. Monthly 59 (1952), 239-241.

12. I. Marx, Problem 4835, Amer. Math. Monthly 67 (1960), 89.

13. A. Erdélyi, W. Magnus, F. Oberhettinger and F. G. Tricomi, Higher transcendental functions, Vol. 1, McGraw-Hill, New York, 1953.

14. J. Surányi, On a problem of old Chinese mathematics, Publ. Math. Debrecen 4 (1956), 195-197.

15. L. Carlitz, Review of On a problem of old Chinese mathematics, Math. Reviews 18 (1957), 4.

\section{MinneAPOLIS, Minnesota}

